\title{
Depresión antenatal y factores relacionados en mujeres primigestas y multigestas
}

\author{
Elena Ovando-Martínez, Paola J. González-Castro*, Ana E. Gutiérrez-Zepeda, Mariana Mendoza-Parra y \\ Daniela Villanueva-Reyes \\ Centro de Tratamiento e Investigación de la Ansiedad, Escuela de Psicología, Universidad de Monterrey. Monterrey, Nuevo León, México
}

\section{Resumen}

Antecedentes: La depresión antenatal es un tema poco estudiado, diagnosticado y tratado; sus consecuencias sobre la madre, el feto y la gestación son graves y con efectos a largo plazo si no se diagnostica y atiende a tiempo. Objetivo: Estudiar la prevalencia de la depresión antenatal en el área metropolitana de Monterrey y su relación con el apoyo social percibido, la autoestima, la ansiedad, así como el número de gestaciones previas. Material y métodos: Estudio de enfoque cuantitativo, diseño transversal, alcance correlacional y comparativo con una muestra de 172 mujeres en el período de gestación. Resultados: Se obtuvo una prevalencia de depresión antenatal del 11.62\%, mientras que los síntomas de ansiedad se registraron en el $16.86 \%$ de las mujeres; el $8.1 \%$ de las gestantes señaló un escaso apoyo social percibido y $5.8 \%$ una autoestima baja. Se hallaron diferencias intergrupales en la variable ansiedad y fueron las mujeres primigestas las que mostraron mayores síntomas de ansiedad. Por último, se encontró que las variables autoestima y ansiedad explican el $67.8 \%$ de la varianza de la variable depresión antenatal.

Palabras clave: Embarazo. Depresión. Ansiedad. Autoestima. Apoyo social.

\section{Antenatal depression and associated factors in primigravid and multigravid women}

\section{Abstract}

Introduction: The phenomena of prenatal depression suffers from a dearth of research interest, being relatively unstudied, and rarely diagnosed or treated. Nonetheless, the consequences for both pregnant mother and fetus can be serious in the absence of diagnosis and appropriate, timely treatment. Objective: To collect demographic data of the prevalence of prenatal depression in the Monterrey metropolitan area and study its relation with prior pregnancies, perceived social support, self-esteem, and anxiety. Material and methods: This qualitative study employs a cross-sectional research design, collecting correlative and comparative data on 174 pregnant women. Results: In the data we obtained, prenatal depression occurs at a prevalence of $11.62 \%$ and anxiety symptomatology in $16.86 \%$, with $8.1 \%$ reporting scarce perceived social support and $5.8 \%$ low self-esteem. Intergroup differences were found between women with multiple pregnancies and those in their first pregnancy who presented higher levels of anxiety. $67.8 \%$ of the variance of antenatal depression was explained by two factors: self-esteem and anxiety.

Key words: Pregnancy. Depression. Anxiety. Self-esteem. Social support.

Correspondencia:

*Paola J. González-Castro

E-mail: paolaj.gonzalez@udem.edu
Disponible en internet: 12-11-2021 Perinatol Reprod Hum. 2020;34(1):3-12 www.perinatologia.mx 0187-5337/C 2021. Instituto Nacional de Perinatología Isidro Espinosa de los Reyes. Publicado por Permanyer. Este es un artículo open access bajo la licencia CC BY-NC-ND (http://creativecommons.org/licenses/by-nc-nd/4.0/). 


\section{Introducción}

La depresión antenatal es un tema poco estudiado, sea en México ${ }^{1}$ e en el mundo entero ${ }^{2}$. Diversos estudios coinciden en que la depresión antenatal se subdiagnostica casi siempre y no se trata 0 se trata de forma insuficiente ${ }^{1-4}$, siendo sus formas más graves las que logran llamar la atención de familiares y especialistas, mientras que sus formas menos agudas pueden imputarse al embarazo mismo². Si bien la depresión en el puerperio se ha estudiado más cuando ésta aparece en el posparto, las cifras demuestran que la depresión antenatal es un trastorno que afecta a un considerable número de mujeres; medida con la Escala de Depresión Posparto de Edimburgo (EDPS), se registra en un $10 \%$ a $12 \%$ de las mujeres gestantes con una incidencia del $3 \%$ al $6 \%$ en su estado mayor ${ }^{2}$; en la población mexicana se han notificado cifras hasta de $23.6 \%$ cuando se utiliza el Inventario de Beck ${ }^{5}$. Al indagar sobre la incidencia de la depresión antenatal según el trimestre que se cursa, se ha encontrado que la incidencia mayor ocurre en el tercer trimestre $(66.6 \%)$, seguido por el primero $(19.1 \%)$ y el segundo $(14.3 \%)^{5}$.

El embarazo implica un cambio profundo para la mujer en las diversas esferas que constituyen su vida: a nivel fisiológico, un cuerpo que se transforma para dar paso a una nueva vida; a nivel cognitivo, el embarazo es también una preparación para asumir un nuevo papel; a nivel psíquico, el embarazo se ha conceptualizado como parteaguas en la psique femenina, en la que de un lado se halla la madre y del otro la mujer ${ }^{6,7}$. Las huellas de la gestación en la estructura cerebral se han rastreado en un estudio fascinante, en el que se comprobaron las modificaciones estructurales que el embarazo inducía en el cerebro de la mujer gestante ${ }^{8}$.

Existen posturas que consideran el embarazo como protector de trastornos psíquicos ${ }^{9}$; se ha constatado también que la incidencia de un episodio psiquiátrico es más elevado durante el primer mes después del posparto que en cualquier otro periodo de la vida e incluso que durante el embarazo, y las mujeres primíparas en posparto son las más expuestas a una admisión psiquiátrica; en cambio, durante el embarazo la posibilidad de un episodio psiquiátrico es menor incluso respecto de las mujeres no embarazadas (las multíparas son quienes tienen más riesgo de un trastorno psiquiátrico $\left.{ }^{10}\right)$. A pesar de este halo protector que confiere el embarazo, la ola de cambios y reajustes que éste trae consigo precipita en algunas mujeres la aparición de diversos padecimientos: depresión, ansiedad y estrés, episodios psicóticos, negación del embarazo, trastornos del comportamiento alimentario, y diversos síntomas menores ${ }^{2}$.

La depresión antenatal se relaciona con un pobre autocuidado materno, una nutrición inadecuada, parto prematuro, anormalidades placentarias, con resultados obstétricos adversos como bajo peso al nacer, morbilidad y mortalidad tanto para la madre como para el feto ${ }^{11-13}$. No tratarla tiene repercusiones adversas a corto y largo plazos, ésta afecta la funcionalidad de la madre, su capacidad para obtener atención prenatal y evitar comportamientos no saludables, factores que a su vez pueden complicar el embarazo ${ }^{3}$. La depresión antenatal, cuando se prolonga al periodo puerperal, implica una menor posibilidad para responder a las necesidades fisiológicas y afectivas del hijo, interacciones maternoinfantiles desadaptadas que pueden tener como consecuencia el maltrato o negligencia, asi como un pobre desarrollo cognitivo ${ }^{13,14}$.

Para el feto, la depresión materna incrementa el ritmo cardiaco y la reactividad fisiológica, al tiempo que el crecimiento es menor; una vez nacido, se ha encontrado que su afecto es mayormente negativo y este afecto puede perdurar a lo largo de la infancia; el desarrollo mental, motor y emocional de los niños con madres deprimidas durante el embarazo es también menor en comparación con niños con madres sin depresión antenatal; esta anomalía materna logra, en el largo plazo, afectar la estabilidad emocional en los años de la infancia ${ }^{13,15}$.

Entre los factores que influyen en la depresión antenatal pueden resaltarse los altos grados de ansiedad ${ }^{16,17}$, el escaso apoyo de la pareja ${ }^{17-20}$, así como la naturaleza multigrávida de la mujer ${ }^{1,10,17}$ y un bajo índice de autoestima ${ }^{20}$. Otros factores se han relacionado como predictores de la depresión antenatal, entre los que se encuentran mala situación económica ${ }^{18,19}$; antecedentes personales psiquiátricos o psicológicos 0 bien antecedentes de depresión antenatal ${ }^{18,19}$; mala salud física, embarazo no planeado y ser ama de casa $^{18}$, así como mayor edad de la mujer gestante, no tener ningún nacido vivo, problemas en embarazos pasados (aborto o muerte neonatal) y violencia doméstica ejercida por cualquier miembro de la familia ${ }^{20,21}$, haber sido víctima de abuso infantil, contar con enfermedades psiquiátricas en los antecedentes familiares, conflictos maritales, episodios estresantes de la vida, ser madre soltera, u autoinfligirse lesiones ${ }^{2,18,19,22}$.

La ansiedad durante el embarazo figura entre los factores de riesgo con mayor efecto por sus consecuencias sobre la madre y el hijo; es un síndrome que revela las inquietudes sobre la salud y el bienestar del 
niño, sobre las experiencias hospitalarias y de salud, así como el parto próximo y sus consecuencias ${ }^{23}$. Los trastornos de ansiedad durante el período perinatal pueden ser más comunes que los de depresión, y cuando se presentan durante la gestación anticipan con frecuencia depresión posparto ${ }^{16,24}$. Las mujeres con grados de ansiedad que interfieren en sus funciones psicológicas y sociales experimentan sufrimiento y dificultades emocionales en los ámbito social, la relación de pareja y el vínculo con el lactante ${ }^{23,25,26}$. La ansiedad durante el embarazo tiene efectos adversos en el neonato debido a la exposición a elevados valores hormonales, en particular cortisol, que contribuyen a un parto prematuro, un riesgo aumentado de bajo peso al nacer, afecciones en el desarrollo neuroconductual en la infancia, retraso en el desarrollo, dificultades para dormir, hipertensión inducida e incremento significativo del riesgo de padecer asma ${ }^{24,27,28}$.

El apoyo social integra todos los recursos del ambiente que ayudan al mantenimiento de las relaciones sociales, que promueven la adaptación y el bienestar del individuo dentro de la sociedad. El apoyo social percibido durante el embarazo tiene influencia positiva sobre la somatización, la angustia y la ansiedad, lo que reduce los grados de depresión y modifica de forma positiva la salud en general de las mujeres gestantes ${ }^{29}$. El apoyo social ofrecido a las gestantes en las primeras etapas del embarazo fortalece la salud de la madre y del recién nacido, y ello influye en el número de controles tempranos de embarazo a los que la mujer se somete, así como en la adopción de conductas saludables $^{29}$. De igual manera, se ha encontrado que el apoyo social altera la salud mental de la gestante y los vínculos positivos materno-infantiles ${ }^{30}$. Desde un punto de vista psicodinámico se ha destacado la importancia de que la mujer cuente con una red de apoyo adecuada, la cual tiene dos funciones principales: la primera consiste en proteger físicamente a la madre, cubrir sus necesidades vitales y retirarla algún tiempo de las exigencias de la realidad externa para que pueda dedicarse a cuidar al hijo; y la segunda, satisfacer la necesidad de la madre de sentirse rodeada, apoyada, acompañada y ayudada durante la crianza, lo que destaca el papel fundamental de la pareja ${ }^{24}$.

La autoestima es la evaluación que una persona hace de sí misma en términos afectivos, ya sea en una valencia negativa o positiva y es a su vez un componente de la autoimagen, la cual se define como la totalidad de pensamientos y sentimientos con los que una persona hace referencia a sí misma ${ }^{31}$. Pocos estudios han investigado el efecto de la autoestima en la depresión materna a pesar de que el embarazo es una etapa que altera la autopercepción de las gestantes y su relación con el mundo y su propio cuerpo ${ }^{20,32,33}$. La diversidad de cambios físicos impulsados por el embarazo, en especial a partir del segundo trimestre, es una razón que puede inducir una modificación en la autopercepción y autovaloración de la gestante. Se ha registrado que las mujeres con alta autoestima tienen una mayor aceptación de los cambios corporales que impone el embarazo, así como de su nuevo papel como madres ${ }^{33}$. Por otro lado, la autoestima muestra un valor predictivo para los síntomas de ansiedad y depresión durante el embarazo ${ }^{20,32}$.

Debido a lo anterior, el objetivo de este estudio fue conocer la prevalencia de la depresión antenatal en el área metropolitana de Monterrey y su relación con la ansiedad, el apoyo social percibido, la autoestima y el número de gestaciones previas. De acuerdo con el propósito de la investigación, se proponen las siguientes hipótesis:

- La depresión antenatal es mayor en el grupo de mujeres multigestas en comparación con el grupo de mujeres primigestas.

- El grado de percepción de apoyo social se relaciona negativamente con la depresión antenatal en ambos grupos.

- El grado de autoestima en la mujer embarazada se relaciona de forma negativa con la depresión antenatal en ambos grupos.

- El grado de ansiedad de la mujer embarazada se relaciona positivamente con la depresión antenatal en ambos grupos.

\section{Método}

Se llevó a cabo un estudio de enfoque cuantitativo, de diseño transversal, con alcance correlacional y comparativo.

\section{Participantes}

Se aplicó la encuesta mediante dispositivos electrónicos a 172 mujeres gestantes, 80 mujeres primigestas $(46.5 \%)$ y 92 multigestas $(53.5 \%)$ cuyo intervalo de edad fue de 20 a 39 años ( $M=27.5$, DS = 5.34) con residencia en el área metropolitana de Nuevo León.

Los criterios de exclusión del estudio fueron que la mujer embarazada padeciera una discapacidad visual o auditiva, que su lugar de residencia no fuera el estado de Nuevo León y que no cumpliera con los criterios de edad previamente estipulados (20 a 40 años). 
Para la obtención de la muestra se distribuyó el cuestionario a través del método de bola de nieve y se asistió a consultas de control prenatal en distintos hospitales públicos y privados de la ciudad de Monterrey y su área metropolitana. Una persona capacitada supervisó en todo momento para resolver las dudas que surgieran en la administración del cuestionario.

\section{Consideraciones éticas}

Se obtuvo el consentimiento informado de las participantes y asimismo se actuó con estricto apego al Código de Ética del Psicólogo ${ }^{34}$, con especial atención a los artículos referentes al respeto de la integridad del individuo (art. 3), a la importancia del secreto profesional (art. 7) y a la necesidad de que toda investigación fuera supervisada por personas técnicamente entrenadas y científicamente calificadas (art. 30).

\section{Instrumentos}

La encuesta constó de cinco instrumentos enfocados en medir la depresión antenatal, la ansiedad, el apoyo social, la autoestima y la recopilación de los datos sociodemográficos.

Escala de depresión posnatal de Edimburgo (EDPE; $\alpha=0.820$ ). Diseñada por Cox, Holden y Sagovsky en $1987^{35}$, validada en la población gestante por Murray y Cox en $1990^{36}$ y validada en México por Alvarado, Sifuentes, Salas y Martínez en el $2006^{3}$, es hoy día el instrumento de tamizaje de depresión perinatal más utilizado en el mundo. Cuenta con 10 reactivos en los que se evalúan los síntomas comunes de un episodio o trastorno depresivo mayor, en los siete días anteriores a la aplicación. El instrumento se contesta mediante una escala tipo Likert de cuatro puntos, del 0 al 3 , en la cual 3 representa muy frecuente; 2 , a veces; 1 , rara vez: y 0 , nunca ${ }^{3}$.

Lista de verificación de síntomas (LVS-90; $\alpha=0.883$ ). La escala evalúa el estrés psicológico o el grado de malestar de la persona en las últimas semanas $^{37}$. Es un instrumento autoaplicable que diseñaron Derogatis, et al. en $1977^{38}$ y validado en México ${ }^{37}$. Para esta investigación se utilizan los reactivos correspondientes a la dimensión de ansiedad, la cual evalúa la presencia de signos generales de ansiedad como nerviosismo, tensión, crisis de pánico y miedos; se contesta de acuerdo con una escala tipo Likert de cinco puntos, en la que 0 equivale a "nada" y 4 a "extremadamente" 37.

Cuestionario de apoyo social funcional (DukeUNC-11; $\alpha=0.907$ ). Mide la percepción del individuo con respecto a la ayuda que los demás le ofrecen. Evalúa el apoyo confidencial, que se define como la posibilidad de contar con personas para comunicarse; y el apoyo afectivo, que se refiere a las demostraciones de amor. Elaborado por Broadhead, Gehlbach, Degruy y Kaplan en $1988^{39}$, lo validaron en la población mexicana en 2007 Piña y Rivera ${ }^{40}$. Contiene 11 reactivos de escala tipo Likert de cinco opciones, en la que 5 representa "Tanto como lo deseo" y 1 "Mucho menos de lo que deseo", y el punto de corte para un apoyo social funcional normal es de 32 puntos.

Escala de autoestima de Rosenberg (EAR; $\alpha=0.807$ ). En el año 1965 se presentó la primera versión de esta prueba, la cual evalúa la valoración que la persona realiza de sí misma en relación con sus cualidades, capacidades, actividades y objetos. La escala se validó en México en $2015^{41}$ y está compuesta por 10 reactivos divididos en dos subescalas, la primera de "autoconfianza" o "satisfacción personal" y la segunda de "autodesprecio" o "devaluación personal".

Cuestionario de datos sociodemográficos. Las investigadoras diseñaron un cuestionario con el fin de obtener información sociodemográfica de las participantes como aspectos generales de la salud, la salud reproductiva y los aspectos específicos de variables que pueden influir en el curso del embarazo.

\section{Análisis de datos}

Se realizó el análisis de datos con el programa SPSS; en función de los objetivos e hipótesis de esta investigación, se llevó a cabo un análisis de correlación de Pearson para evaluar la relación entre las variables; una prueba $T$ de Student para analizar las diferencias intergrupales entre primigestas y multigestas determinó si el número de embarazos era un factor de riesgo para la depresión perinatal o sus factores adjuntos. Por último, se llevó a cabo un análisis de regresión lineal para determinar las variables que predicen con más efectos la depresión en el periodo antenatal.

\section{Resultados}

\section{Resultados descriptivos}

En cuanto a los datos sociodemográficos, las participantes señalaron en su mayoría ser casadas (68\%) o vivir en unión libre (19.8\%). Un 41.9\% indicó ser ama de casa y la misma cantidad ser empleada. La escolaridad concluida de la muestra fue licenciatura (29.7\%), preparatoria (23.8\%) y secundaria (21.5\%). El $68.6 \%$ de 
Tabla 1. Datos sociodemográficos de las participantes

\begin{tabular}{|c|c|c|c|}
\hline & Total & Primigestas & Multigestas \\
\hline n & 172 & 80 & 92 \\
\hline Edad & $\begin{array}{c}\text { Rango }=20 \text { a } 39 \\
(\mathrm{M}=27.5 ; \mathrm{DT}=5.34)\end{array}$ & $\begin{array}{c}\text { Rango }=20 \text { a } 36 \\
(\mathrm{M}=24.45 ; \mathrm{DT}=3.97)\end{array}$ & $\begin{array}{l}\text { Rango }=20 \text { a } 39 \text { años } \\
(\mathrm{M}=30.15 ; \mathrm{DT}=4.96)\end{array}$ \\
\hline Estado civil & $\begin{array}{c}\text { Casada }=117(68 \%) \\
\text { Unión libre }=34(19.8 \%) \\
\text { Soltera }=11(6.4 \%) \\
\text { Con pareja }=9(5.2 \%) \\
\text { Divorciada }=1(0.6 \%)\end{array}$ & $\begin{array}{c}\text { Casada }=51(63.75 \%) \\
\text { Unión libre }=16(20 \%) \\
\text { Soltera }=7(8.75 \%) \\
\text { Con pareja }=6(7.5 \%) \\
\text { Divorciada }=0\end{array}$ & $\begin{array}{c}\text { Casada }=66(71.73 \%) \\
\text { Unión libre }=18(19.56 \%) \\
\text { Soltera }=4(4.37 \%) \\
\text { Con pareja }=3(3.26 \%) \\
\text { Divorciada }=1(1.08 \%)\end{array}$ \\
\hline Ocupación & $\begin{array}{c}\text { Ama de casa }=72(41.9 \%) \\
\text { Empleada }=72(41.9 \%) \\
\text { Desempleada }=1(.58 \%) \\
\text { Comerciante }=3(1.7 \%) \\
\text { Empresaria }=10(5.8 \%) \\
\text { Estudiante }=10(5.8 \%) \\
\text { Otro }=4(2.3 \%)\end{array}$ & $\begin{array}{c}\text { Ama de casa }=23(28.75 \%) \\
\text { Empleada }=36(45 \%) \\
\text { Desempleada }=1(1.25 \%) \\
\text { Comerciante }=1(1.25 \%) \\
\text { Empresaria }=7(8.75 \%) \\
\text { Estudiante }=9(11.25 \%) \\
\text { Otro }=3(3.75 \%)\end{array}$ & $\begin{array}{c}\text { Ama de casa }=49(53.26 \%) \\
\text { Empleada }=36(39.13 \%) \\
\text { Desempleada }=0 \\
\text { Comerciante }=2(2.17 \%) \\
\text { Empresaria }=3(8.75 \%) \\
\text { Estudiante }=1(3.26 \%) \\
\text { Otro }=1(3.26 \%)\end{array}$ \\
\hline Estudios concluidos & $\begin{aligned} \text { Primaria } & =9(5.2 \%) \\
\text { Secundaria } & =37(21.55) \\
\text { Preparatoria } & =41(23.8 \%) \\
\text { Bachillerato técnico } & =1(0.6 \%) \\
\text { Carrera técnica } & =21(12.2 \%) \\
\text { Licenciatura } & =51(29.7 \%) \\
\text { Posgrado } & =12(7 \%)\end{aligned}$ & $\begin{array}{c}\text { Primaria }=0 \\
\text { Secundaria }=11(13.7 \%) \\
\text { Preparatoria }=25(31.2 \%) \\
\text { Bachillerato técnico }=1(1.2 \%) \\
\text { Carrera técnica }=11(13.7 \%) \\
\text { Licenciatura }=28(35 \%) \\
\text { Posgrado }=4(5 \%)\end{array}$ & $\begin{array}{c}\text { Primaria }=9(5.2 \%) \\
\text { Secundaria }=26(21.55) \\
\text { Preparatoria }=16(23.8 \%) \\
\text { Bachillerato técnico }=0 \\
\text { Carrera técnica }=10(12.2 \%) \\
\text { Licenciatura }=23(29.7 \%) \\
\text { Posgrado }=8(7 \%)\end{array}$ \\
\hline Estatus socioeconómico & $\begin{array}{c}\text { Bajo }=5(2.9 \%) \\
\text { Medio-bajo }=14(8.1 \%) \\
\text { Medio }=118(68.6 \%) \\
\text { Medio-alto }=32(18.6 \%) \\
\text { Alto }=3(1.7 \%)\end{array}$ & $\begin{array}{c}\text { Bajo }=1(1.2 \%) \\
\text { Medio-bajo }=5(6.2 \%) \\
\text { Medio }=51(63.7 \%) \\
\text { Medio-alto }=21(26.2 \%) \\
\text { Alto }=2(2.5 \%)\end{array}$ & $\begin{array}{c}\text { Bajo }=4(4.3 \%) \\
\text { Medio-bajo }=9(9.8 \%) \\
\text { Medio }=67(72.8 \%) \\
\text { Medio-alto }=11(11.9 \%) \\
\text { Alto }=1(1 \%)\end{array}$ \\
\hline
\end{tabular}

DT: Desviación estándar; M: Media;

la población refirió tener un estado socioeconómico medio y el $18.6 \%$ uno medio-alto (Tabla 1 ).

El $47 \%$ de la muestra se integró con mujeres primigestas y el $53 \%$ con multigestas. Del total de la muestra, el $39.5 \%$ cursaba el segundo trimestre y el $60.5 \%$ el tercero. Se encontró que un $18 \%$ de la muestra señaló la pérdida de un hijo en el embarazo, y estas pérdidas ocurrieron en su mayor parte en el primer trimestre (15.1\%); el 1.7\% informó la pérdida durante el parto y el $1.2 \%$ durante el primer año de vida. El diagnóstico del embarazo actual de las gestantes se comunicó como normal en el $77 \%$ de los casos, de alto riesgo en un $11.6 \%$ y con diabetes gestacional en el $8.1 \%$. El $10 \%$ de las mujeres embarazadas registró tener el diagnóstico de alguna afección médica crónica. Por otra parte, un $7.6 \%$ ha tenido un diagnóstico previo de depresión y un $4.1 \%$ uno psicológico o psiquiátrico (Tabla 2).

Del total de la muestra de mujeres gestantes se encontró que el $11.62 \%$ de las gestantes sufre probablemente depresión antenatal; el $8.1 \%$ señala un escaso apoyo social percibido y el $16.86 \%$ de las mujeres registró síntomas de ansiedad. En cuanto a los índices de autoestima, el $5.8 \%$ de las mujeres embarazadas registró una autoestima baja, el $7.6 \%$ de las gestantes una autoestima media y $86.6 \%$ una autoestima adecuada.

Se llevó a cabo una comparación entre las puntuaciones en la escala EDPE, en la cual se encontró que no hay diferencia significativa entre las primigestas $(\mathrm{M}=7.087$; $\mathrm{DT}=4.39)$ y las multigestas $(\mathrm{M}=6.554$; $\mathrm{DT}=4.818), t(172)=-0.754 ; p=0.402$, lo que rechaza así la primera hipótesis. Al realizar el mismo análisis para las demás escalas, no se encontró una diferencia significativa intergrupal para el apoyo percibido $t(172)=-0.149 ; p=0.142$, ni para los síntomas de ansiedad $t(172)=-1.935 ; p=0.154$, a pesar lo cual los índices de ansiedad fueron mucho más elevados en las primigestas $(21.25 \% ; M=11.712 ; \mathrm{DT}=8.83)$ que en las multigestas (13.04\%; $M=13.04 \% ; M=9.271$; DT $=7.707)$. Una diferencia moderadamente significativa se encontró al comparar las medias de la escala de autoestima entre los grupos ( $t(172)=1.452$; 
Tabla 2. Datos sobre la salud de las participantes

\begin{tabular}{|c|c|c|c|}
\hline & Total & Primigestas & Multigestas \\
\hline$n$ & 172 & 80 & 92 \\
\hline $\begin{array}{l}\text { Trimestre } \\
\text { de gestación }\end{array}$ & $\begin{array}{c}2^{\circ}=68(39.5 \%) \\
3^{\circ}=104(60.5 \%)\end{array}$ & $\begin{array}{l}2^{\circ}=33(41.25 \%) \\
3^{\circ}=47(58.75 \%)\end{array}$ & $\begin{array}{l}2^{\circ}=35(38 \%) \\
3^{\circ}=57(62 \%)\end{array}$ \\
\hline $\begin{array}{l}\text { Diagnóstico } \\
\text { del embarazo }\end{array}$ & $\begin{array}{c}\text { Normal = } 133(77.3 \%) \\
\text { Alto riesgo }=20(11.6 \%) \\
\text { Diabetes gest. }=14(8.1 \%) \\
\text { Anemia }=2(1.2 \%) \\
\text { Complicaciones con la placenta } \\
\text { o líquido amniótico }=2(1.2 \%)\end{array}$ & $\begin{array}{c}\text { Normal }=69(86.2 \%) \\
\text { Alto riesgo }=5(6.2 \%) \\
\text { Diabetes gest. }=3(3.7 \%) \\
\text { Anemia }=2(2.5 \%) \\
\text { Complicaciones con la placenta } \\
\text { o líquido amniótico }=1(1.25 \%)\end{array}$ & $\begin{array}{c}\text { Normal }=64(69.6 \%) \\
\text { Alto riesgo }=15(16.3 \%) \\
\text { Diabetes gest. }=11(11.95 \%) \\
\text { Anemia }=0 \\
\text { Complicaciones con la placenta } \\
\text { o líquido amniótico }=1(1 \%)\end{array}$ \\
\hline $\begin{array}{l}\text { Diagnóstico } \\
\text { patología crónica }\end{array}$ & $\begin{aligned} \text { No } & =155(90 \%) \\
\text { Sí } & =17(10 \%)\end{aligned}$ & $\begin{aligned} \text { No } & =71(89 \%) \\
\text { Sí } & =9(11 \%)\end{aligned}$ & $\begin{array}{c}\text { No }=84(91.3 \%) \\
\text { Sí }=8(8.7 \%)\end{array}$ \\
\hline $\begin{array}{l}\text { Diagnóstico } \\
\text { Depresión }\end{array}$ & $\begin{array}{c}\text { No }=159(92.4 \%)) \\
\text { Sí }=13(7.65)\end{array}$ & $\begin{array}{c}\text { No }=74(92.5 \%) \\
\text { Sí }=6(7.5 \%)\end{array}$ & $\begin{array}{c}\text { No }=85(92.4 \%) \\
\text { Sí }=7(7.6 \%)\end{array}$ \\
\hline $\begin{array}{l}\text { Otro Diagnóstico } \\
\text { Psicol. o psiq. }\end{array}$ & $\begin{array}{c}\text { No }=165(65.9 \%) \\
\text { Sí }=7(4.1 \%)\end{array}$ & $\begin{array}{c}\text { No }=76(95 \%) \\
\text { Sí }=4(5 \%)\end{array}$ & $\begin{array}{c}\text { No }=89(96.7 \%) \\
\text { Sí }=3(3.3 \%)\end{array}$ \\
\hline
\end{tabular}

$p=0.084)$ y fue el grupo de las primigestas el que registró un índice más alto de autoestima (91.25\%; $\mathrm{M}=35.28$; $\mathrm{DT}=4.42)$ en comparación con el grupo de multigestas $(83.43 \% ; M=34.22 ; D T=5.05)$.

\section{Análisis de correlaciones}

Se realizó un análisis de correlación de Pearson entre las distintas variables del estudio, el cual arrojó una correlación negativa entre la variable de depresión y las variables de apoyo social $(r=-0.288 ; p \leq 0.01)$ y autoestima $(r=-0.511 ; p \leq 0.01)$, y a partir de estos resultados se aceptaron las hipótesis segunda y tercera planteadas. Finalmente, la hipótesis cuarta se acepta al encontrar en los resultados una correlación positiva entre la depresión antenatal y la ansiedad $(r=0.560 ; p \leq 0.01)$; la correlación positiva se observa también cuando se ha tenido un diagnóstico previo de depresión ( $r=0.222$; $p \leq 0.01$ ) o cuando se ha perdido a un hijo durante el primer año de vida $(r=0.169$; $p \leq 0.05$ ) (Tabla 3).

\section{Análisis de regresión}

Por último, se realizó un análisis de regresión para muestras independientes con la muestra global a fin de determinar el efecto de las variables ansiedad, autoestima y apoyo social percibido sobre la depresión antenatal. En el modelo propuesto se encontró que las variables autoestima y ansiedad explican el $67.8 \%$ de la varianza de la variable depresión antenatal (Tabla 4).

\section{Discusión}

La prevalencia encontrada de depresión antenatal en el área metropolitana de Nuevo León fue de $11.62 \%$, cifra que concuerda con estudios en los que la prevalencia de la depresión antenatal se halla entre el 10\% $y$ el $15 \%$ de las mujeres gestantes ${ }^{3-5}$.

Se identificó que un $8.1 \%$ de las gestantes percibe un escaso apoyo social; esta cifra es menor a los hallazgos de otros estudios realizados en México y Latinoamérica en los que se observa que $12.3 \%$ al $18 \%$ de las mujeres gestantes señalan un escaso o nulo apoyo emocional en este periodo ${ }^{42}$.

La incidencia de síntomas de ansiedad en las mujeres gestantes fue de $16.86 \%$, cifra que concuerda con otros resultados de investigaciones realizadas en México en las que se registran síntomas de ansiedad de $9 \%$ a $15 \%$ según sea el trimestre en el que se encuentra la mujer ${ }^{24}$. En este estudio se advirtió que en el segundo trimestre la ansiedad se presentó en $17.64 \%$ de las mujeres y en el tercero en el $16.34 \%$.

Los grados de autoestima durante el embarazo se han estudiado en la población adolescente, a diferencia de los resultados encontrados; cuando se investiga esta variable en la población adolescente se reconoce un elevado porcentaje de la población con grados bajos de autoestima y éstos varían de acuerdo con las escalas utilizadas. La Escala de Autoestima de Rosenberg registra bajos grados de autoestima en un $31.82 \%$ de la población adolescente ${ }^{43}$. Los estudios sobre la autoestima en gestantes no adolescentes son escasos, 
Tabla 3. Correlaciones entre las variables

\begin{tabular}{|c|c|c|c|c|c|c|c|}
\hline & & $\begin{array}{l}\text { Depresión } \\
\text { antenatal }\end{array}$ & $\begin{array}{l}\text { Apoyo } \\
\text { Social } \\
\text { Percibido }\end{array}$ & $\begin{array}{l}\text { Síntomas } \\
\text { de } \\
\text { Ansiedad }\end{array}$ & Autoestima & $\begin{array}{l}\text { Diagnósticoprevio } \\
\text { de depresión }\end{array}$ & $\begin{array}{l}\text { Pérdida de un } \\
\text { hijo durante el } \\
\text { primer año de } \\
\text { vida }\end{array}$ \\
\hline Depresión antenatal & $\begin{array}{l}\text { Pearson's r } \\
\text { p-value }\end{array}$ & & $\begin{array}{l}-0.288^{* *} \\
<0.01\end{array}$ & $\begin{array}{l}0.566^{* *} \\
<0.01\end{array}$ & $\begin{array}{l}-0.511^{* *} \\
<0.01\end{array}$ & $\begin{array}{l}0.222^{* *} \\
<0.01\end{array}$ & $\begin{array}{l}0.169^{*} \\
<0.05\end{array}$ \\
\hline $\begin{array}{l}\text { Apoyo Social } \\
\text { Percibido }\end{array}$ & $\begin{array}{l}\text { Pearson's r } \\
\text { p-value }\end{array}$ & $\begin{array}{l}-0.288^{* *} \\
<0.01\end{array}$ & & $\begin{array}{l}-0.123 \\
0.109\end{array}$ & $\begin{array}{l}0.514^{* *} \\
<0.01\end{array}$ & $\begin{array}{l}-0.052 \\
0.495\end{array}$ & $\begin{array}{l}0.063 \\
0.410\end{array}$ \\
\hline $\begin{array}{l}\text { Síntomas de } \\
\text { Ansiedad }\end{array}$ & $\begin{array}{l}\text { Pearson's r } \\
\text { p-value }\end{array}$ & $\begin{array}{l}0.566^{* *} \\
<0.01\end{array}$ & $\begin{array}{l}-0.123 \\
0.109\end{array}$ & & $\begin{array}{l}-0.266^{* *} \\
<0.01\end{array}$ & $\begin{array}{l}0.158^{*} \\
<0.05\end{array}$ & $\begin{array}{l}0.073 \\
0.340\end{array}$ \\
\hline Autoestima & $\begin{array}{l}\text { Pearson's r } \\
\text { p-value }\end{array}$ & $\begin{array}{l}-0.511^{* *} \\
<0.01\end{array}$ & $\begin{array}{l}0.514^{* *} \\
<0.01\end{array}$ & $\begin{array}{l}-.266^{* *} \\
<0.01\end{array}$ & & $\begin{array}{l}-0.112 \\
0.142\end{array}$ & $\begin{array}{l}-0.130 \\
0.089\end{array}$ \\
\hline $\begin{array}{l}\text { Diagnóstico previo } \\
\text { de depresión }\end{array}$ & $\begin{array}{l}\text { Pearson's r } \\
\text { p-value }\end{array}$ & $\begin{array}{l}0.222^{* *} \\
<0.01\end{array}$ & $\begin{array}{c}-0.052 \\
.495\end{array}$ & $\begin{array}{l}0.158^{*} \\
<0.05\end{array}$ & $\begin{array}{l}-0.112 \\
0.142\end{array}$ & & $\begin{array}{l}-0.031 \\
0.686\end{array}$ \\
\hline $\begin{array}{l}\text { Pérdida de un hijo } \\
\text { durante el primer año } \\
\text { de vida }\end{array}$ & $\begin{array}{l}\text { Pearson's r } \\
\text { p-value }\end{array}$ & $\begin{array}{l}0.169^{*} \\
<0.05\end{array}$ & $\begin{array}{l}0.063 \\
0.410\end{array}$ & $\begin{array}{l}.073 \\
.340\end{array}$ & $\begin{array}{l}-0.130 \\
0.089\end{array}$ & $\begin{array}{l}-0.031 \\
0.686\end{array}$ & \\
\hline
\end{tabular}

Tabla 4. Análisis de regresión para las variables Depresión antenatal, autoestima y ansiedad

\begin{tabular}{|c|c|c|c|c|c|c|}
\hline & $\beta$ & Stand Error & Beta Stand Coef & $t$ & $p$ value & $\mathrm{R}^{2}$ \\
\hline $\begin{array}{l}\text { Escala de Depresión Prenatal de Edimbu } \\
\text { Model } 1 \\
\text { (Constant) } \\
\text { Escala de autoestima de Rosenberg } \\
\text { SCL-90 Ansiedad }\end{array}$ & $\begin{array}{c}17.129 \\
-0.374 \\
0.257\end{array}$ & $\begin{array}{l}2.097 \\
0.057 \\
0.033\end{array}$ & $\begin{array}{c}-0.388 \\
0.463\end{array}$ & $\begin{array}{l}8.168 \\
-6.617 \\
7.894\end{array}$ & $\begin{array}{l}0.001 \\
0.001 \\
0.001\end{array}$ & 0.460 \\
\hline
\end{tabular}

en contraste con los bajos grados de autoestima en el embarazo adolescente; este estudio halló que el $86.6 \%$ de las mujeres indicó una autoestima elevada.

En esta investigación se encontró que no hay diferencias significativas entre las puntuaciones de posible depresión de las primigestas y multigestas, por lo cual la primera hipótesis se rechazó; estos resultados coinciden con otras investigaciones ${ }^{44}$.

En cuanto a la segunda hipótesis, se planteó que existe una relación negativa entre la depresión antenatal y el apoyo social percibido, hipótesis que se confirmó y que coincide con los resultados de otros estudios $^{29}$. Asimismo, en un estudio se establecieron cuatro componentes de apoyo social, como la necesidad de apoyo, la satisfacción con la ayuda recibida, la cantidad de personas disponibles para ayuda y su red de conflicto, que influyeron en el estrés y la depresión materna ${ }^{45}$. La comparación de la percepción del apoyo social entre el grupo de las primigestas y las multigestas no mostró diferencias significativas; este resultado también coincide con previas investigaciones en las que se ha estudiado la calidad de la relación con la madre y la pareja, y comparado a primigestas y multigestas, cuyos resultados tampoco encontraron diferencias intergrupales significativas ${ }^{46}$.

La tercera hipótesis establece que existe una relación entre la ansiedad y la depresión, la cual fue aceptada; cuanta más ansiedad presenta la mujer gestante, mayor incidencia de depresión tendrá. Se ha informado que la ansiedad eleva el riesgo de padecer depresión perinatal en un $3.85 \%$, y cuando la gestante tiene una acumulación de episodios estresantes y ansiedad grave este riesgo aumenta hasta $10.3 \%{ }^{47}$. De la misma manera, se ha señalado que la ansiedad durante el embarazo se encuentra dentro de los factores de riesgo con mayor efecto en las consecuencias para la madre y el hijo ${ }^{23}$. Al igual que los resultados de los autores, otros estudios han observado también que las mujeres primigestas muestran mayores grados de ansiedad en el periodo de gestación ${ }^{48}$ y que este grupo 
es el que tiene mayores sentimientos de incertidumbre y por ende mayor ansiedad. La ansiedad materna, durante la segunda mitad del embarazo, puede inducir cambios en el sistema de adaptación al estrés manejado por el niño; es por ello que es importante detectar a tiempo los trastornos de ansiedad durante el embarazo ${ }^{28}$. Por otro lado, la personalidad es una poderosa influencia en la manera en que las mujeres hacen frente a las demandas de la maternidad, en las cuales las madres ansiosas son más vulnerables a la angustia emocional, ya que pueden sentirse aún más ansiosas cuando no están conformes con las expectativas de los demás ${ }^{49}$. Asimismo, en la comparación del nivel de ansiedad entre grupos se encontró en este estudio que las primigestas tienen mayor grado de ansiedad que las multigestas, contrario a lo que otros autores han observado $^{12}$.

La última hipótesis postula que el grado de autoestima de la mujer embarazada se relaciona con la depresión antenatal; esta relación fue negativa, es decir, a menor grado de autoestima, mayor depresión. Esto coincide con otros resultados encontrados en los que se afirma que durante el embarazo ocurren distintos cambios, tanto físicos como psicológicos, que favorecen un posible terreno para la depresión y la baja autoestima ${ }^{50}$.

Como resultados adicionales se observó que existe una relación negativa entre la edad y la ansiedad de las embarazadas, así como una relación negativa al momento de comparar la semana de embarazo y la percepción de apoyo social; sin embargo, no se encontraron estudios que confirmaran los resultados de esta investigación; es importante concederle más relevancia a esta información en futuros estudios.

Por otro lado, se identificó una relación positiva entre la semana de embarazo y el grado de autoestima de la gestante, por lo que cuantas más semanas de embarazo tenga la mujer, mayor será su autoestima. En este sentido, algunos autores han encontrado que, con el curso del embarazo, la mujer se ve y se siente floreciente y ello eleva sus grados de autoestima, pero también se ha resaltado el hecho de que durante los últimos meses los movimientos propios del hijo funcionan como un supresor de la angustia, una señal de que avanza bien ${ }^{6}$.

En este estudio, la ansiedad y la autoestima son variables que no sólo se relacionan con la depresión antenatal, sino que pueden explicar el advenimiento de ésta hasta en un $67.8 \%$. Distintos estudios coinciden con que la variable autoestima tiene un valor predictivo sobre los síntomas de ansiedad y depresión en el embarazo $^{32}$, mientras que para otros es uno de los principales factores de riesgo para el desarrollo de depresión antenatal ${ }^{18}$. De la misma manera, se ha encontrado el valor predictivo de la ansiedad sobre el desarrollo de la depresión antenatal ${ }^{16,23,24}$.

\section{Conclusiones}

Los resultados hallados en esta investigación son relevantes y buscan dar más notoriedad a la problemática de la depresión antenatal en México. Con base en los resultados de esta investigación se pueden desarrollar programas de intervención primaria mediante técnicas psicoeducativas que difundan las principales características, factores de riesgo y protectores de la depresión antenatal, con especial énfasis en las variables de autoestima y apoyo social percibido, identificadas como sólidos predictores de la depresión antenatal.

La prevalencia de depresión antenatal encontrada en este estudio es sin duda elevada, si se tienen en cuenta las consecuencias que esta alteración puede traer en el embarazo y la salud física y psicológica del niño y su madre en el posparto. La EDPE es un instrumento de tamizaje que, en virtud de la facilidad de uso e interpretación, pueden utilizar los especialistas de la salud (médicas, enfermeras, parteras, trabajadoras sociales) durante las citas de control prenatal.

Se recomienda a los especialistas de la salud que atienden a esta población poner especial atención a los cambios psicológicos que experimenta la mujer embarazada; y, ante la sospecha de una probable depresión antenatal, debe realizarse una entrevista y canalizar con un especialista para confirmar el diagnóstico de tal manera que la mujer pueda recibir un tratamiento adecuado.

La evaluación y el control psicológico de la depresión antenatal de la mujer embarazada no deben omitirse o aislarse de la consulta de control prenatal, dadas las graves consecuencias para la madre y el recién nacido, los efectos en el embarazo y la probabilidad de padecer depresión posparto ${ }^{25-28}$.

El periodo gestacional es un momento ideal para tratar la depresión antenatal, tanto por la cercanía de la mujer gestante a los sistemas de salud como por las escasas barreras reconocidas en este periodo para un tratamiento psicológico ${ }^{9}$; de esta manera, la gestación ofrece a los profesionales de salud un terreno fértil para el estudio y el diseño de intervenciones tempranas que logren contrarrestar los efectos de la depresión sobre la madre y su hijo en el posparto, al tiempo 
que se logran abatir los altos costos que tienen en los sistemas de salud las depresiones no tratadas'.

Entre las limitantes de este estudio debe mencionarse que la muestra, debido a sus particularidades, se obtuvo por medio del método de bola de nieve. Otra limitación representa el hecho de que, al ser los instrumentos autoinformes, es posible que se presente el fenómeno de deseabilidad social, lo que afecta las respuestas de las mujeres en función de un deseo de aceptación. A pesar de estas limitantes, los resultados de este estudio son concluyentes en cuanto a la necesidad de continuar la investigación en esta población mediante estudios longitudinales a fin de desarrollar y evaluar métodos de prevención oportuna de la depresión antenatal para esta población.

\section{Financiamiento}

Este trabajo no contó con financiamiento alguno.

\section{Conflicto de intereses}

Las autoras declaran que no tienen conflicto de intereses para la divulgación del manuscrito.

\section{Responsabilidades éticas}

Protección de personas y animales. Los autores declaran que los procedimientos seguidos se conformaron a las normas éticas del comité de experimentación humana responsable y de acuerdo con la Asociación Médica Mundial y la Declaración de Helsinki.

Confidencialidad de los datos. Los autores declaran que han seguido los protocolos de su centro de trabajo sobre la publicación de datos de pacientes.

Derecho a la privacidad y consentimiento informado. Los autores han obtenido el consentimiento informado de los pacientes o sujetos referidos en el artículo. Este documento obra en poder del autor de correspondencia.

\section{Bibliografía}

1. Berenzon S, Lara MA, Robles R, Medina-Mora ME. Depresión: estado del conocimiento y la necesidad de políticas públicas y planes de acción en México. Salud Pública Mex. 2013:55:74-80.

2. Dayan J, Andro G, Dugnat M. Psychopathologie de la périnatalité et de la parentalité. Elsevier Masson, 2014.

3. Alvarado-Esquivel C, Sifuentes-Álvarez A, Salas-Martínez C. Validation of the Edinburgh Postpartum Depression Scale in a population of adult pregnant women in Mexico. J Clin Med Res. 2014;6:374-8.

4. Jadresic ME. Depresión perinatal: detección y tratamiento. Rev Médica Clínica Las Condes 2014;25:1019-1028.

5. Ruiz-Acosta GJ, Organista-Gabriel H, López-Avendaño RA, Cortázar-Ramírez L, Vinalay-Carrillo I. Prevalencia de depresión en embarazadas de una institución de salud de primer nivel. Salud y Adm. 2017;4:25-35.
6. Lemoine-Luccioni E. La partición de las mujeres. Amorrortu Editores, 1982.

7. Brousse MH. Femme ou mère. La Cause Freudienne 1993;24:30-33.

8. Hoekzema E. Pregnancy leads to long-lasting changes in human brain structure. Nat Neurosci. 2017;20:287-296.

9. Bydlowski M. Le regard intérieur de la femme enceinte, transparence psychique et représentation de l'objet interne. Devenir 2001;13:41.

10. Paffenberg R. Epidemiological aspects of mental illness associated whit childbearing. In: Motherhood and mental illness. Brockington I, Kumar R, ed. Academic Press, 1982.

11. Milgrom J, Gemmill AW. Screening for perinatal depression. Best Pract Res Clin Obstet Gynaecol. 2014;28:13-23.

12. Urdaneta J. Prevalencia de depresión posparto en primigestas y multíparas valoradas por la escala de Edimburgo. Rev Chil Obs Ginecol 2010;75:312-320.

13. Field $T$, Diego $M$, Hernández-Reif M. Prenatal depression effects on the fetus and newborn: a review. Infant Behav Dev. 2006;29:445-455.

14. Stein A. Effects of perinatal mental disorders on the fetus and child. Lancet 2014;384:1800-1819.

15. Marr M. 3.20 The trajectory of maternal prenatal stress is associated with newborn amygdala functional connectivity and infant negative affect development over the first two years of life. J Am Acad Child Adolesc. Psychiatry 2018;57:S188.

16. Coelho HF, Murray L, Royal-Lawson M, Cooper PJ. Antenatal anxiety disorder as a predictor of postnatal depression: a longitudinal study. J Affect Disord. 2011;129:348-353.

17. Garfield L. Depressive symptoms in the second trimester relate to low oxytocin levels in African-American women: a pilot study. Arch Womens Ment Health. 2015;18:123-129.

18. Bavle AD. Antenatal depression in a tertiary care hospital. Indian J Psychol. Med. 2016;38:31-5.

19. Ali DF, Butt DA, Hameed DA, Furqan DA, Ali F. Antenatal depression; frequency and factors associated in patients presenting in tertiary care hospital. Prof Med J. 2017;24:458-461.

20. Sung Hee L, Ja Jung $E$. Antenatal depression among women of advanced maternal age: self-esteem and spousal support as predicting factors. Int Inf Inst (Tokyo) Inf. 2016;19:5857-5872.

21. Ali NS, Azam IS, Ali BS, Tabbusum G, Moin SS. Frequency and associated factors for anxiety and depression in pregnant women: a hospital-based cross-sectional study. Sci World J. 2012.

22. Burns A. A pilot randomised controlled trial of cognitive behavioural therapy for antenatal depression. BMC Psychiatry. 2013;13.

23. Dunkel Schetter C, Tanner L. Anxiety, depression and stress in pregnancy. Curr Opin Psychiatry. 2012;25:141-148.

24. Navarrete LE, Lara-Cantú MA, Navarro C, Gómez ME, Morales F. Factores psicosociales que predicen síntomas de ansiedad posnatal y su relación con los síntomas depresivos en el posparto. Rev Investig Clínica. 2012;64:625-633.

25. de la Cruz Góngora Rodríguez P, Pantí Simá SA. Ansiedad durante el embarazo. Desarro Científico Enferm. 2010;18:289-292.

26. Stadtlander L. Anxiety and pregnancy. Int J Childbirth Educ. 2017;32:32-34.

27. Andersson L, Sundström-Poromaa I, Wulff M, Åström M, Bixo M. Implications of antenatal depression and anxiety for obstetric outcome. Obstet Gynecol. 2004;104:467-476.

28. Glover V. Maternal depression, anxiety and stress during pregnancy and child outcome; what needs to be done. Best Pract Res Clin Obstet Gynaecol. 2014;28:25-35

29. Guarino L, Scremín F, Borrás S. Nivel de información y apoyo social como predictoras de la salud y calidad de vida durante el embarazo. Psychol Av Discip. 2013;7:13-21.

30. Myors KA, Schmied, V, Johnson M, Cleary M. 'My special time': australian women's experiences of accessing a specialist perinatal and infant mental health service. Health Soc Care Community. 2014;22:268-277.

31. Rosenberg M. Society and the adolescent self-image. Princeton University Press, 1965.

32. Franck $E$. The role of self-esteem instability in the development of postnatal depression: a prospective study testing a diathesis-stress account. J Behav Ther Exp Psychiatry. 2016;50:15-22.

33. Bezerra-Cavalcante JC, Carvalho de Sousa VE, de Oliveira Lopes MV. Baixa autoestima situacional em gestantes: uma análise de acurácia. Rev Bras Enferm. 2012;65:977-983.

34. Sociedad Mexicana de Psicología. Código Ético del Psicólogo. Trillas, 2002.

35. Cox JL, Holden JM, Sagovsky R. Detection of postnatal depression. Development of the 10-item Edinburgh Postnatal Depression Scale. Br J Psychiatry. 1987;150:782-6.

36. Murray D, Cox JL. Screening for depression during pregnancy with the edinburgh depression scale (EDDS). J Reprod Infant Psychol. 1990; 8:99-107.

37. Cruz-Fuentes C, López-Bello L, Blas-García C, González-Macías L, Chávez-Balderas RA. Datos sobre la validez y confiabilidad de la Symptom Check List 90 (SCL90) en una muestra de sujetos mexicanos. Salud Ment. 2005;28:72-81. 
38. Derogatis LR. Symptom Checklist-90 Revised (SCL-90-R). Minneapolis: NCS Assessments, 1992.

39. Broadhead WE, Gehlbach SH, de Gruy FV, Kaplan BH. The Duke-UNC Functional Social Support Questionnaire. Measurement of social support in family medicine patients. Med Care. 1988;26:709-23.

40. Piña-López AJ, Rivera-Icedo BM. Validación del cuestionario de apoyo social funcional en personas seropositivas al vih del noroeste de México. Cienc y Enfermería. 2007:13:53-63

41. Jurado-Cárdenas D, Jurado-Cárdenas S, López-Villagrán $K$, Querevalú Guzmán B. Validez de la Escala de Autoestima de Rosenberg en universitarios de la Ciudad de México. Rev Latinoam Med Conduct Lat Am J Behav. 2015;5:18-22.

42. Lara MA. Síntomas depresivos en el embarazo y factores asociados en pacientes de tres instituciones de salud de la ciudad de México. Salud Ment. 2006;29:55-62.

43. Ceballos-Ospino GA, Camargo-Goenaga K, Jiménez-Sánchez I, Requena-Mendoza K. Artículos de investigación. Nivel de autoestima en adolescentes embarazadas en la comuna 5 de Santa Marta (Colombia). Rev Psicol Univ Antioquia. 2011;3:29-38.
44. Carrillo-Pino FF. Prevalencia de la depresión en gestantes Quechua hablantes a través de la escala de Edimburgo adaptada al Quechua. Universidad Nacional de San Antonio Abad del Cusco, 2013.

45. Martínez-Schallmoser L, Telleen S, Macmullen NJ. The effect of social support and acculturation on postpartum depression in mexican american women. J Transcult Nurs. 2003;14:329-338.

46. Armengol-Asenjo R, Chamarro-Lusar A, Teresa-García-Dié Muñoz M. Aspectos psicosociales en la gestación: el Cuestionario de Evaluación Prenatal. An Psicol. 2007;23:25-32.

47. Vinaccia S, Sánchez MV, Bustamante EM, Cano EJ, Tobón S. Conducta de enfermedad y niveles de depresión en mujeres embarazadas con diagnóstico de preeclampsia. Int J Clin Heal Psychol. 2006;6:41-51.

48. Ontiveros-Pérez M, López-España JT. Adaptación psicosocial y niveles de ansiedad en embarazadas primigestas de bajo riesgo obstétrico. Bol Clin Hosp Infant Edo Son. 2010;27:834881847.

49. Hanley J. Perinatal mental health: a guide for health professionals and users. Wiley-Blackwell, 2009.

50. Alderete-Jiménez L. Autoestima y depresión en adolescentes embarazadas. Desarro Cientif Enferm 2012;20. 